\title{
Distinct Topological Surface States on the Two Terminations of $\mathrm{MnBi}_{4} \mathrm{Te}_{7}$
}

\author{
Xuefeng Wu, ${ }^{1, *}$ Jiayu Li๑,${ }^{1, *}$ Xiao-Ming Ma๑, ${ }^{1, *}$ Yu Zhang $\odot,{ }^{1,2, *}$ Yuntian Liu, ${ }^{1}$ Chun-Sheng Zhou, ${ }^{1}$ Jifeng Shao, ${ }^{1}$ \\ Qiaoming Wang, ${ }^{1}$ Yu-Jie Hao, ${ }^{1}$ Yue Feng, ${ }^{1}$ Eike F. Schwier, ${ }^{3}$ Shiv Kumar, ${ }^{3}$ Hongyi Sun, ${ }^{1}$ Pengfei Liu, ${ }^{1}$ Kenya Shimada@, ${ }^{3}$ \\ Koji Miyamoto, ${ }^{3}$ Taichi Okuda, ${ }^{3}$ Kedong Wang, ${ }^{1}$ Maohai Xie $\odot,{ }^{2}$ Chaoyu Chen $\odot,{ }^{1}$ \\ Qihang Liu $\odot,{ }^{1,4, \dagger}$ Chang Liu $\odot,{ }^{1, \$}$ and Yue Zhao $\oplus^{1, \S}$ \\ ${ }^{1}$ Shenzhen Institute for Quantum Science and Engineering and Department of Physics, \\ Southern University of Science and Technology, Shenzhen 518055, China \\ ${ }^{2}$ Department of Physics, The University of Hong Kong, Hong Kong, China \\ ${ }^{3}$ Hiroshima Synchrotron Radiation Center, Hiroshima University, 2-313 Kagamiyama, \\ Higashi-Hiroshima 739-0046, Japan \\ ${ }^{4}$ Guangdong Provincial Key Laboratory for Computational Science and Material Design, \\ Southern University of Science and Technology, Shenzhen 518055, China
}

(Received 6 February 2020; revised 15 April 2020; accepted 5 May 2020; published 16 July 2020)

\begin{abstract}
The recently discovered intrinsic magnetic topological insulator $\mathrm{MnBi}_{2} \mathrm{Te}_{4}$ has been met with unusual success in hosting emergent phenomena such as the quantum anomalous Hall effect and the axion insulator states. However, the surface-bulk correspondence of the Mn-Bi-Te family, composed by the superlatticelike $\mathrm{MnBi}_{2} \mathrm{Te}_{4} /\left(\mathrm{Bi}_{2} \mathrm{Te}_{3}\right)_{n}(n=0,1,2,3 \ldots)$ layered structure, remains intriguing but elusive. Here, by using scanning tunneling microscopy and angle-resolved photoemission spectroscopy techniques, we unambiguously assign the two distinct surface states of $\mathrm{MnBi}_{4} \mathrm{Te}_{7}(n=1)$ to the quintuple-layer (QL) $\mathrm{Bi}_{2} \mathrm{Te}_{3}$ termination and the septuple-layer (SL) $\mathrm{MnBi}_{2} \mathrm{Te}_{4}$ termination, respectively. A comparison of the experimental observations with theoretical calculations reveals diverging topological behaviors, especially the hybridization effect between the QL and SL, on the two terminations. We identify a gap on the QL termination, originating from the hybridization between the topological surface states of the QL and the bands of the SL beneath, and a gapless Dirac-cone band structure on the SL termination with time-reversal symmetry. The quasiparticle interference patterns further confirm the topological nature of the surface states for both terminations, continuing far above the Fermi energy. The QL termination carries a spin-helical Dirac state with hexagonal warping, while at the SL termination, a strongly canted helical state from the surface lies between a pair of Rashba-like splitting bands from its neighboring layer. Our work elucidates an unprecedented hybridization effect between the building blocks of the topological surface states and also reveals the termination-dependent time-reversal symmetry breaking in a magnetic topological insulator.
\end{abstract}

DOI: $10.1103 /$ PhysRevX.10.031013

\section{INTRODUCTION}

The recent discovery of the intrinsic magnetic topological insulator $\mathrm{MnBi}_{2} \mathrm{Te}_{4}$ and its derivatives $\mathrm{MnBi}_{2} \mathrm{Te}_{4} /\left(\mathrm{Bi}_{2} \mathrm{Te}_{3}\right)_{n}(n=1,2, \ldots)$, comprising alternating

\footnotetext{
${ }^{*}$ These authors contributed equally to this work.

${ }^{\dagger}$ To whom correspondence should be addressed. liuqh@ sustech.edu.cn.

ॠTo whom correspondence should be addressed. liuc@sustech .edu.cn

${ }^{\S}$ To whom correspondence should be addressed. zhaoy@ sustech.edu.cn

Published by the American Physical Society under the terms of the Creative Commons Attribution 4.0 International license. Further distribution of this work must maintain attribution to the author(s) and the published article's title, journal citation, and DOI.
}

Subject Areas: Condensed Matter Physics, Magnetism, Topological Insulators

layers of $\mathrm{MnBi}_{2} \mathrm{Te}_{4}$ and nonmagnetic topological insulator (TI) $\mathrm{Bi}_{2} \mathrm{Te}_{3}$, has boosted exciting possibilities of producing exotic quantum phenomena by engineering topology and magnetism at the atomic scale [1-10]. Specifically, $\mathrm{MnBi}_{2} \mathrm{Te}_{4}$, having antiferromagnetic (AFM) order and, thus, broken time-reversal symmetry, holds the potential for realizing both a quantum anomalous Hall insulator and an axion insulator [11-13]. When subjected to an external magnetic field of around 5-10 T, few-layer $\mathrm{MnBi}_{2} \mathrm{Te}_{4}$ turns to a ferromagnetic (FM) quantum anomalous Hall insulator $[11,12]$. On the other hand, a zero Hall plateau is observed in even-layer $\mathrm{MnBi}_{2} \mathrm{Te}_{4}$ as an indicator for the axion insulator [13]. However, recent angle-resolved photoemission spectroscopy (ARPES) measurements show a robust surface Dirac cone, indicating that time-reversal symmetry is preserved at the surface of $\mathrm{MnBi}_{2} \mathrm{Te}_{4}$ [14-17]. Other members in van der Waals $\mathrm{MnBi}_{2} \mathrm{Te}_{4} /\left(\mathrm{Bi}_{2} \mathrm{Te}_{3}\right)_{n}$, with 
(a)

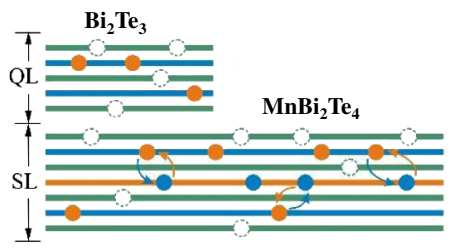

(b)
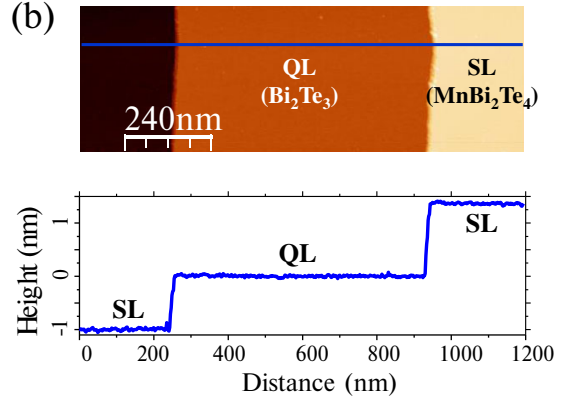

$\mathrm{Bi}_{2} \mathrm{Te}_{3} \mathrm{QL}$ termination

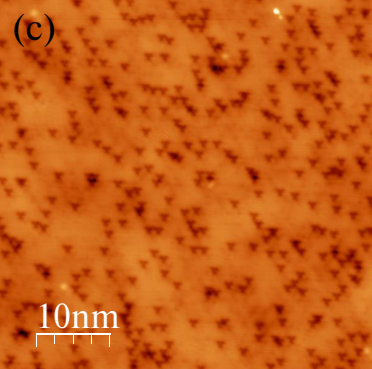

(d)

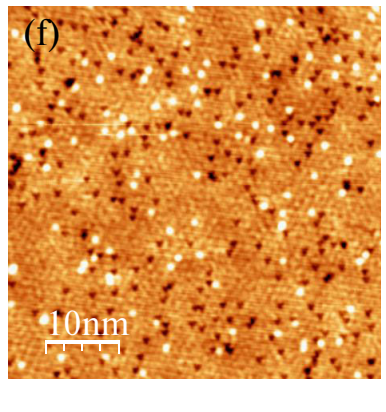

$\mathrm{MnBi}_{2} \mathrm{Te}_{4} \mathrm{SL}_{\text {termination }}$

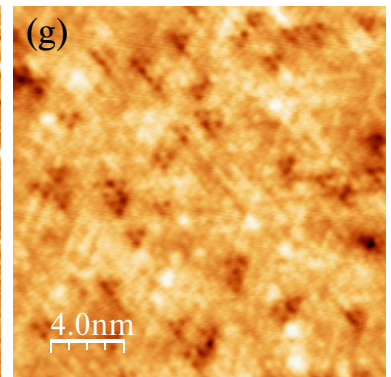

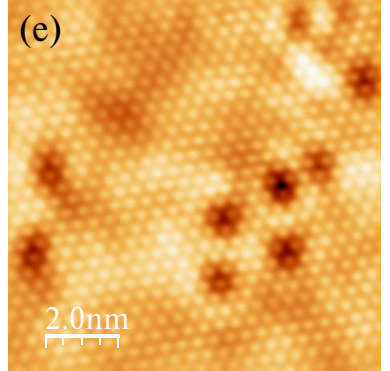

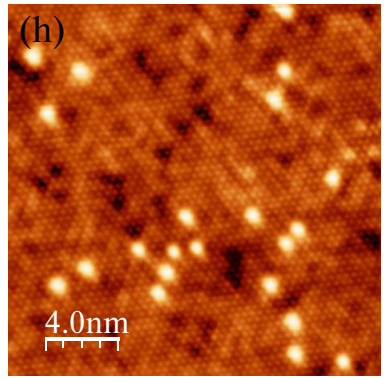

FIG. 1. STM topographic images of the $\mathrm{MnBi}_{4} \mathrm{Te}_{7}$ surface. (a) Schematic diagram of two terminations- the QL termination of $\mathrm{Bi}_{2} \mathrm{Te}_{3}$ and the SL termination of $\mathrm{MnBi}_{2} \mathrm{Te}_{4}$ - with atomic defects. (b) A large area topographic image of $\mathrm{MnBi}_{4} \mathrm{Te}_{7}$ surface showing two kinds of terrace steps. The lower part of (b) shows a line profile with step heights of 1.01 and $1.35 \mathrm{~nm}$ along the blue line, for the QL termination of $\mathrm{Bi}_{2} \mathrm{Te}_{3}$ and the $\mathrm{SL}$ termination of $\mathrm{MnBi}_{2} \mathrm{Te}_{4}$, respectively. A considerable number of defects can be found in the enlarged views of the QL (c) and SL (f) terminations. (d),(e) and (g),(h) are the atomic resolution images of the same areas at different biases on the QL and SL terminations, respectively. The dark defects are found on both QL and SL terminations. For the SL, an additional type of defect is observed as a bright dot at both positive and negative biases. Tunneling parameters: (b) $V_{\text {bias }}=1.2 \mathrm{~V}$, and $I_{t}=20 \mathrm{pA}$; (c),(f) $V_{\text {bias }}=1.0 \mathrm{~V}$ and $I_{t}=20 \mathrm{pA}$; (d) $V_{\text {bias }}=0.5 \mathrm{~V}$ and $I_{t}=1 \mathrm{nA}$; (e) $V_{\text {bias }}=-1 \mathrm{~V}$ and $I_{t}=1 \mathrm{nA} ;(\mathrm{g}) V_{\text {bias }}=0.5 \mathrm{~V}$ and $I_{t}=200 \mathrm{pA}$; (h) $V_{\text {bias }}=-1 \mathrm{~V}$ and $I_{t}=200 \mathrm{pA}$.

tunable interlayer magnetic exchange coupling, also exhibit controversially a gap or gapless feature at different terminations [18-25], highlighting the complication of the interplay between magnetism and topology and the crucial role of defects and disorder in such a material system.

Surface-bulk correspondence is generally considered to be the unifying feature relating the topology of the bulk bands to the surface states in topological materials [26]. However, this correspondence is intriguing in the superlatticelike $\mathrm{MnBi}_{2} \mathrm{Te}_{4} /\left(\mathrm{Bi}_{2} \mathrm{Te}_{3}\right)_{n}$ layered structure for $n \geq 1$, where the hybrid structure creates distinct electronic structures on separate terminations due to the interplay of topology and magnetism at the interfaces of magnetic TI $\left(\mathrm{MnBi}_{2} \mathrm{Te}_{4}\right)$ and nonmagnetic TI $\left(\mathrm{Bi}_{2} \mathrm{Te}_{3}\right)$ layers. Local probe approaches to the scattering processes [27-29] on different terminations of the van der Waals heterostructure are crucial to determine the spin configuration of the topological surface states and advance the theoretical understanding of heterostructure engineering. Moreover, despite the evidence of the band structure in $\mathrm{MnBi}_{2} \mathrm{Te}_{4} /\left(\mathrm{Bi}_{2} \mathrm{Te}_{3}\right)_{n}$ series from ARPES and transport studies, so far there has been little experimental work on probing the robustness of these states against scattering
[30,31], which is one of the key properties toward the application of topological devices.

Here, we present the first local probe scanning tunneling microscopy (STM) measurements of $\mathrm{MnBi}_{4} \mathrm{Te}_{7}(n=1)$ in both the real and momentum spaces. Combined with the observed ARPES band dispersion, we unambiguously assign the observed electronic band structures to the $\mathrm{MnBi}_{2} \mathrm{Te}_{4}$ septuple-layer (SL) and $\mathrm{Bi}_{2} \mathrm{Te}_{3}$ quintuple-layer (QL) terminations. Together with the input of ARPES (both regular and spin-resolved) and theoretical calculations, further investigation on the scattering process by quasiparticle interference (QPI) patterns unveils the spin configuration of their topological surface states. Unlike the surface state of a conventional TI (e.g., $\mathrm{Bi}_{2} \mathrm{Se}_{3}$ ) that is simply localized at the surface layer with attenuation into the bulk, the surface states of $\mathrm{MnBi}_{4} \mathrm{Te}_{7}$ show strong hybridization between the SL and QL at both terminations. For QL termination, a gap is formed due to the hybridization between the topological surface bands of the topmost QL and the bands from the neighboring SL. For SL termination, our results suggest a restoration of timereversal symmetry, possibly due to the magnetically disordered surface. The spin texture of the topological surface 
bands shows a pair of Rashba-like splitting bands from the QL underneath and a strongly warped band from the SL, the hybridization of which contributes to the flower-shaped QPI patterns. Our findings of such diverging topological behaviors on the two terminations of $\mathrm{MnBi}_{4} \mathrm{Te}_{7}$ strongly rely on the hybridization of bands from different building blocks of magnetic TI and nonmagnetic TI, providing new insights into the surface-bulk correspondence of magnetic TIs and guidance to heterostructure engineering in the emerging intrinsic magnetic topological systems.

\section{SURFACE TOPOGRAPHY}

Figure 1 illustrates the topographic images obtained by STM, where the two terminations are identified unambiguously. As shown in Fig. 1(b), the crystal naturally cleaves at the QL termination of $\mathrm{Bi}_{2} \mathrm{Te}_{3}$ and the SL termination of $\mathrm{MnBi}_{2} \mathrm{Te}_{4}$ with step heights of 1.01 and $1.35 \mathrm{~nm}$, respectively. Comparing the enlarged views of the QL [Fig. 1(c)] and SL [Fig. 1(f)] terminations, many more surface defects can be found on the SL termination. Even on the surface defect-free area, QL termination shows less corrugation, indicating lower bulk defect beneath the topmost layer (Fig. S2 [32]). Two types of major defects are found on the SL surface, categorized as bright and dark spots, the former of which is almost absent on the QL surface. We then obtain the atomic resolution images of the Teterminated QL [Figs. 1(d) and 1(e)] and SL [Figs. 1(g) and 1(h)] surfaces at different bias voltages. Similar bright spots are also found in the STM study of $\mathrm{MnBi}_{2} \mathrm{Te}_{4}$ [33]. On both terminations, the dark defects merge from triangularly placed holes to one triangular dark spot when the bias voltage is shifted from 0.5 to $-1 \mathrm{~V}$, while the atoms from the topmost layer remain intact. Right on top of the dark defects on both terminations, as shown in Fig. S2 [32], there is a slight rightward shift (approximately $10 \mathrm{meV}$ ) of $d I / d V$ spectra compared with the defect-free area, indicating a local $p$-type doping from this local defect. Such topographic and spectroscopic behaviors were previously seen on the surface of Mn-doped $\mathrm{Bi}_{2} \mathrm{Te}_{3}$ [34,35], where it is attributed to be substitutional Mn atoms close to the sample surface. This similarity, especially on the QL with limited types of surface defects, implies the possibility of magnetic atom migration from the designed sites in the middle of the SL. The density of such defects is about $3.0 \%$ on the QL termination and $2.1 \%$ on the SL termination. Note that these results are for the topmost and the second atomic layer, as STM is surface sensitive. The possibility of Mn migration may induce complicated surface magnetic order or disorder on both terminations, especially for the QL, as stated in previous studies that such an amount of magnetic dopant is sufficient enough to induce long-range ferromagnetic order in magnetically doped topological insulators $[28,36,37]$.

\section{ELECTRONIC STRUCTURES}

We now focus on the defect-free regions to obtain our averaged $d I / d V$ spectra by scanning tunneling spectroscopy (STS). The results, as shown in Fig. 2(a), reveal a great suppression of density of states (DOS) from approximately -285 to $-350 \mathrm{meV}$ on the QL termination, differing from the $\mathrm{V}$-shaped, Dirac-like suppression of the DOS near approximately $-285 \mathrm{meV}$ on the SL termination. The SL surface holds a higher density of states above $-285 \mathrm{meV}$, while, on the other side (below approximately $-400 \mathrm{meV}$ ), the QL surface hosts higher DOS. Although it is not straightforward to determine gaps or positions of bands solely from the STS spectra, we can compare with our ARPES data to gain a better understanding of the states. Figures 2(c) and 2(d) are two representative laser- $\mu$-ARPES spectra obtained from the same batch of crystals [38], representing the electronic structure on the two surface terminations of $\mathrm{MnBi}_{4} \mathrm{Te}_{7}$. In Fig. 2(c), the outer electron pocket at $\bar{\Gamma}$ is seen to have a relatively flat bottom, where an apparent gaplike suppression of spectral weight is observed between $E=-270$ to $-320 \mathrm{meV}$, in agreement with the STS at the QL termination [marked by the purple and the red arrows in Figs. 2(a) and 2(c)]. Much higher ARPES intensity is observed for the bands below $E=-400 \mathrm{meV}$, which is also reproduced by the high DOS below $-400 \mathrm{meV}$ bias voltage in the STS. We thus conclude that Fig. 2(c) shows the band structure of the QL termination. Similar analysis also reveals that Fig. 2(d) depicts the bands of the SL termination, where a high DOS is observed from $E_{F}$ to $E=-0.3 \mathrm{eV}$. In this energy region, the bottom of one of the conduction bands at $E=-150 \mathrm{meV}$ can be identified in the STS by a DOS peak [brown arrows in Figs. 2(a) and 2(d)]. Moreover, the $\mathrm{X}$-shaped, Dirac-like linear dispersion and the gapless crossing point at $E=-285 \mathrm{meV}$ [red arrow in Fig. 2(d)] is endorsed by a V-shaped dip at the SL STS whose minimum locates at approximately $-280 \mathrm{meV}$ [red arrow in Fig. 2(a)]. Therefore, by comparing with the STS spectra, we experimentally ascertain the assignment of the QL and SL terminations in the ARPES measurements for the first time, which is also confirmed by our surface-dependent DOS calculations from density functional theory (DFT) [see Fig. 2(a)].

We also perform DFT calculations of the band dispersion for $\mathrm{MnBi}_{4} \mathrm{Te}_{7}$ slabs to advance our understanding of the two terminations. For the QL termination, we assume that the local moment of the SLs near the surface remains the same as that in the bulk, i.e., $A$-type AFM with out-of-plane spin orientation confirmed by neutron diffraction measurements [39]. The resulting band structure in Fig. 2(e) shows that the Dirac cone of the surface states is gapped by the magnetic proximity from the second SL, merging into the valence band at approximately $400 \mathrm{meV}$ below the Fermi level [see the green box in Fig. 2(e)]. More importantly, an indirect gap near the Dirac point energy occurs. The layer 

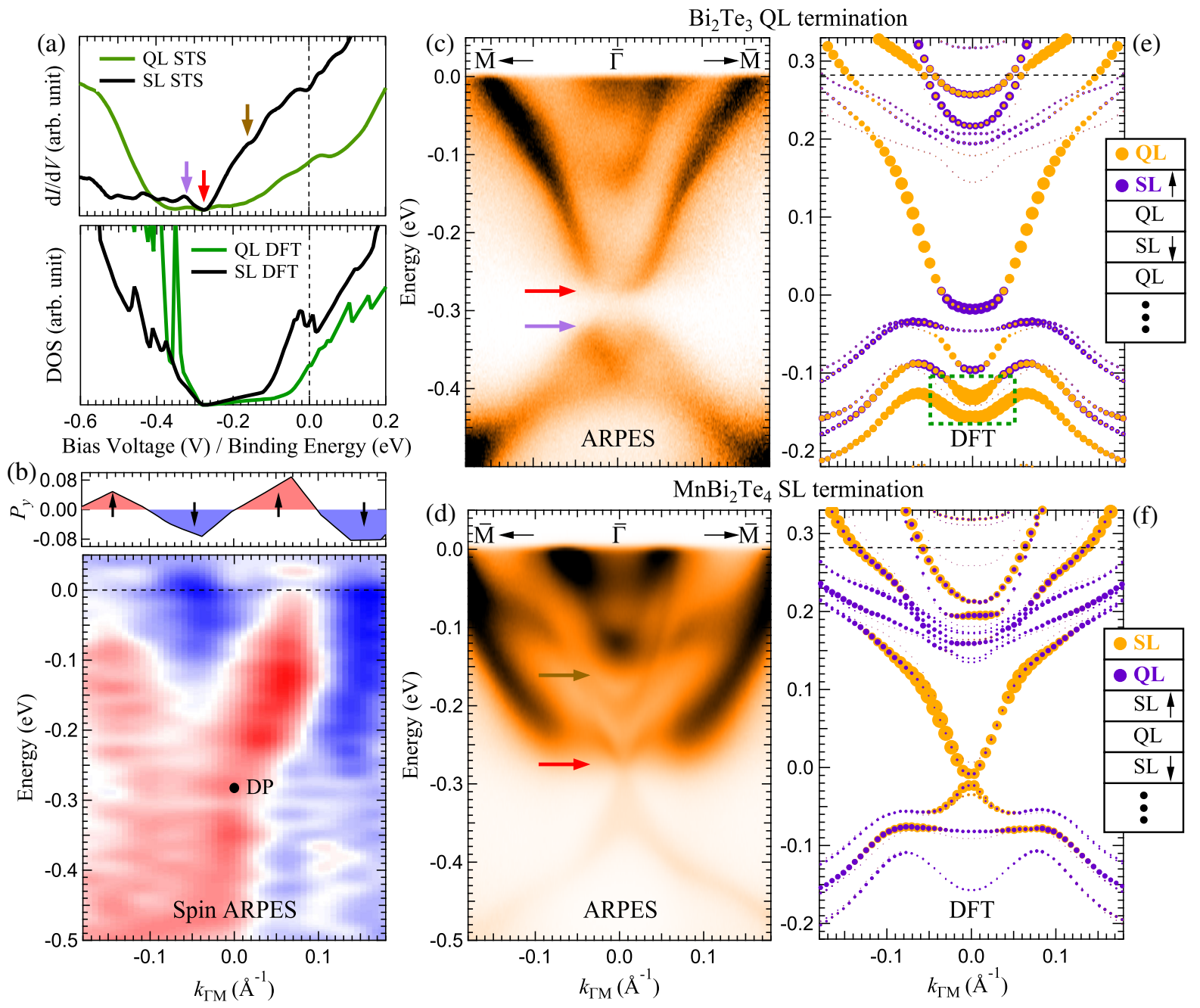

FIG. 2. Electronic structure of the two terminations of $\mathrm{MnBi}_{4} \mathrm{Te}_{7}$. (a) Top: Averaged $d I / d V$ spectra of a series of STS spectra obtained at defect-free areas of the QL (green) and SL (black) terminations. Tunneling parameters: $V_{\text {bias }}=0.5 \mathrm{~V}$ and $I_{t}=500 \mathrm{pA}$. The arrows mark the hybridization gap opening on the QL termination (red and purple), the Dirac point (red), and the onset of the inner Rashba-like splitting band on the SL termination (brown), in good agreement with the ARPES measurement. Bottom: DFT-calculated surfacedependent DOS for both terminations. The surface projections include the top two surface layers, containing one SL and one QL. The calculated DOS plots are offset to $-285 \mathrm{meV}$ to match the Dirac point of SL termination. The dashed line marks the Fermi level. (b) Spin-polarized ARPES map for both terminations of $\mathrm{MnBi}_{4} \mathrm{Te}_{7}$, with a line cut at $E_{B}=0.08 \mathrm{eV}$ showing the antiparallel helicity of the two bands. (c) and (d) are the ARPES intensity maps of the QL and SL terminations at the $\bar{\Gamma}-\bar{M}$ direction, respectively. Arrows highlight important STS features in (a). (e) and (f) show the results from density functional theory calculations for the QL and SL terminations, respectively. The slab model assumes a nonmagnetic SL termination for (f), while for (e) an A-type AFM spin configuration is used with the assumption that the magnetic order of the second SL is protected by the topmost QL. The green dashed box in (e) marks the gapped Dirac cone of the surface states.

projection of the Bloch wave functions clearly shows that there is a band inversion between the conduction and valence bands, which is dominated by the topmost QL and the second SL, respectively [see Fig. S3(a) [32]]. Therefore, the indirect gap is caused by the hybridization effect between these two layers. This result is in sharp contrast to the conventional TI or magnetic-doped TI, where the surface states around the Fermi level are governed by the surface layer, with attenuation into the bulk. It is also worth noting that other ARPES studies on the QL termination reveal a $\Lambda$-shaped intensity within the above mentioned hybridization gap [21,22]. With the assumption of an A-type AFM spin configuration, such an in-gap state can be interpreted as a hybridization effect between different orbitals contributed by the surface QL and its neighboring SL, respectively.

For the $\mathrm{MnBi}_{2} \mathrm{Te}_{4}$ SL termination [Fig. 2(f)], the experimental $A$-type AFM order with an out-of-plane moment will inevitably open a sizable gap of around $60 \mathrm{meV}$ at the surface state [Fig. S4(a) [32] ], inconsistent with our ARPES observation showing a gapless Dirac cone. In real materials, surface magnetism can be different 
from the bulk due to various reasons including surface reconstruction, disorder, or spin canting, etc., [40-42], which essentially affects the magnetic-related topological behaviors. In $\mathrm{MnBi}_{4} \mathrm{Te}_{7}$, a considerable number of defects, as spotted on both terminations, may further complicate the surface magnetism. In principle, several possible magnetic orders on the surface, such as in-plane ferromagnetism or disordered local moments, could lead to a vanishing surface gap [see Figs. S4(b) and S4(c) [32] ] [14]. Without loss of generality, we suggest a topmost nonmagnetic SL with disordered spin to be the scenario, giving rise to a restoration of the time-reversal symmetry on the SL termination. As shown in Fig. 2(f), by assuming a nonmagnetic SL surface layer, our DFT calculations obtain almost linear dispersion with a negligibly gapped Dirac cone (gap size less than $10 \mathrm{meV}$ ), originating from the proximity effect of the third $\mathrm{MnBi}_{2} \mathrm{Te}_{4}$ layer, in good agreement with our ARPES results [Fig. 2(d)]. Unlike the QL termination, the projection onto layers shows that the linear Dirac bands are mainly contributed by the surface SL [Fig. S3(b) [32] ].

\section{SPIN-SELECTIVE QUASIPARTICLE INTERFERENCE}

Having established the distinct band dispersion at the two terminations, now we carry out Fourier transform scanning tunneling spectroscopy (FT STS) at various energies to further investigate the topological surface states. The disorder scattering mixes the states with different moments $\left(\boldsymbol{k}_{1}\right.$ and $\boldsymbol{k}_{2}$ ) at the same contour of constant energy (CCE) for the surface bands, resulting in a standing wave with wave vector $\left(\boldsymbol{k}_{1}-\boldsymbol{k}_{2}\right) / 2$. Such interference patterns can be probed as a local DOS modulation in the real space with wavelength $\lambda=2 \pi / q$, where $\boldsymbol{q}=\boldsymbol{k}_{1}-\boldsymbol{k}_{2}$ [43,44]. In the simplest approach, the quasiparticle interference patterns should match the joint density of states (JDOS) as a function of momentum differences $(\boldsymbol{q})$ between the two scattering states, $\operatorname{JDOS}(\boldsymbol{q}, E)=\int I(\boldsymbol{k}, E) I(\boldsymbol{k}+\boldsymbol{q}, E) d^{2} \boldsymbol{k}$, where $I(\boldsymbol{k})$ can be experimentally determined from CCEs in the ARPES measurement. Figures 3(c) and 3(d) show typical CCEs of ARPES data for the QL and SL terminations, respectively, at approximately $-50 \mathrm{meV}$. Comparing with the calculated JDOS patterns from ARPES CCE maps [Figs. 3(e) and 3(f)], the FT STS maps [Figs. 3(i) and 3(j)] display clear suppression of scattering intensity along $\bar{\Gamma}-\bar{K}$ directions. Previous FT STS studies on topological insulators suggest that the scattering process can be greatly affected by the spin texture of the topological surface states, as helicity allows interference to occur only when there is a finite spin overlap between the two scattering states $[27,28,35,45]$. On the QL termination, a hexagonally warped shape of surface band structure is observed [Fig. 3(c)], accompanied by suppressed $\bar{\Gamma}-\bar{K}$ scattering in the QPI pattern. This scattering resembles the warping-induced attenuation of $\bar{\Gamma}-\bar{K}$ scattering in
$\mathrm{Bi}_{2} \mathrm{Te}_{3}$, where an out-of-plane spin component can be introduced in between the warped corners, suggesting that the spin texture on QL termination can be understood in a similar manner.

Compared with the case of the QL termination, the QPI pattern of the SL is more intricate. We first look into the results from spin-resolved ARPES for possible spin texture guidance at the SL termination. Figure 2(b) represents a spin resolved ARPES map of $\mathrm{MnBi}_{4} \mathrm{Te}_{7}$ along the $\bar{\Gamma}-\bar{M}$ direction measured with a high-efficiency very low energy electron diffraction (VLEED) spin detector [46], showing the spin component along the tangential direction of the CCE band contours. The data are a mixed result of both the SL and QL terminations due to the millimeter-sized spatial resolution of the spin ARPES device. Interestingly, the electronic states of $\mathrm{MnBi}_{4} \mathrm{Te}_{7}$ show an updown-up-down spin configuration above the Dirac point energy $(-280<E<0 \mathrm{meV})$, resembling a typical Rashbalike spin splitting. We conclude such a spin pattern comes from the SL termination for the following reasons: (i) The $k$ space locations of the spin-polarized bands in Fig. 2(b) match those for the SL termination [Fig. 2(d)] to a better degree than those for the QL termination [Fig. 2(c)]. Specifically, the spin pattern at moderate binding energies (i.e., $E_{b} \sim 0.2 \mathrm{eV}$ ) spreads to a wider range of $k_{\Gamma M}$ than the QL bands do [dashed curves in Figs. 2(b) and 2(d)]. (ii) The inner ring in the QL termination [Fig. 3(c)] is assigned as a bulk electronic state [15], where spin polarization is assumed to be far less polarized than that of surface states. Therefore, our spinresolved ARPES results suggest a pair of Rashba-like splitting bands on the SL termination, which could originate from the potential gradient of the confining electrostatic potential in the surface space-charge regions [47].

Taking the above-mentioned experimental observations and DFT calculations into account, we construct a uniform surface $k \cdot p$ model Hamiltonian to extract the central information of the electronic structures of both terminations, including Dirac bands, Rashba bands, hexagonal warping [48], hybridization between the SL and QL, and the exchange field due to the proximity effect from the bulk magnetism. It reads in the following:

$$
H_{k}=\tau_{+} H_{k}^{1}+\tau_{-} H_{k}^{2}+\Delta \tau_{x} .
$$

In Eq. (1), $H_{\boldsymbol{k}}^{1}=\varepsilon_{\boldsymbol{k}}^{D}+v_{F}^{D}(\boldsymbol{\sigma} \times \boldsymbol{k})_{z}+(\lambda / 2)\left(k_{+}^{3}+k_{-}^{3}\right) \sigma_{z}$ describes a gapless, warped Dirac cone representing both the topmost $\mathrm{Bi}_{2} \mathrm{Te}_{3}$ layer for the $\mathrm{QL}$ termination and the topmost $\mathrm{MnBi}_{2} \mathrm{Te}_{4}$ layer with a disordered local moment for the SL termination $\left(k_{ \pm}=k_{x} \pm i k_{y}\right) . \quad H_{\boldsymbol{k}}^{2}=\varepsilon_{\boldsymbol{k}}^{R}+$ $v_{F}^{R}(\boldsymbol{\sigma} \times \boldsymbol{k})_{z}+m_{z}^{R} \sigma_{z}$ describes a pair of Rashba bands with possible proximity-induced exchange field $m_{z}^{R}$ capturing the dispersion from the second topmost layers for both terminations. $\varepsilon_{k}^{D / R}$ and $v_{F}^{D / R}$ denote the parabolic dispersion and Fermi velocity of Dirac and Rashba bands, respectively, with $\lambda$ and $\Delta$ the parameters of hexagonal warping and 


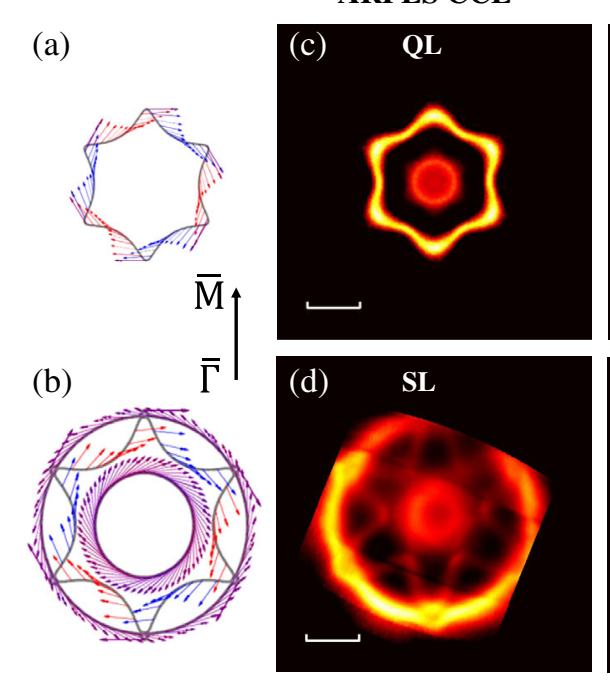

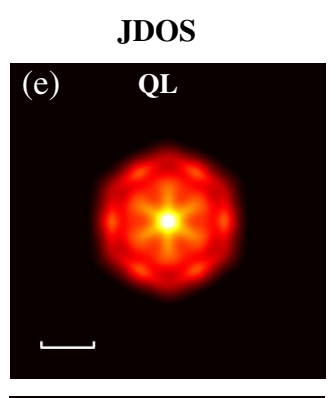

Spin-selective JDOS
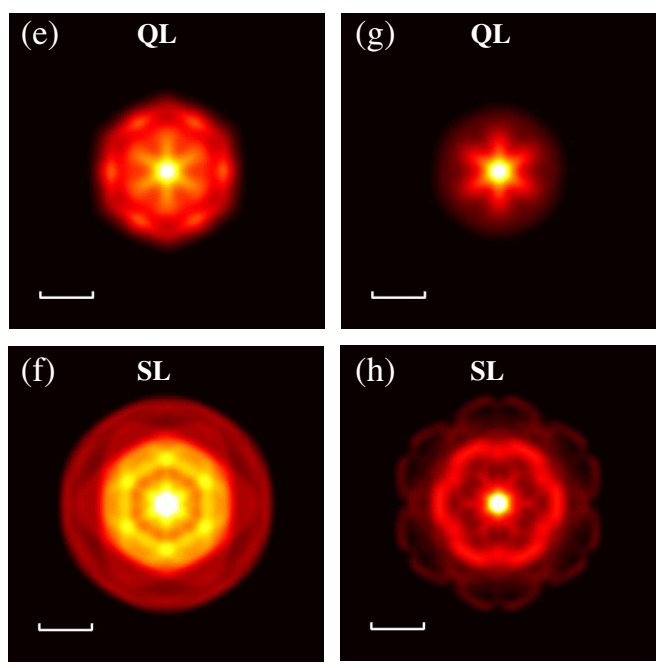

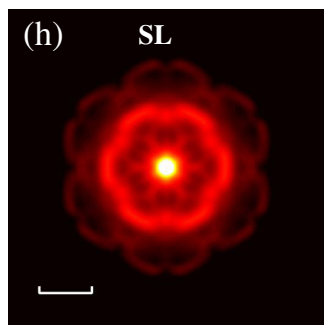

FT STS
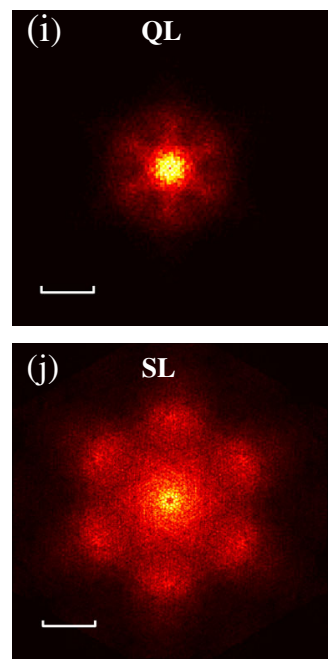

FIG. 3. ARPES contours of constant energy, JDOS, and spin-selective JDOS calculated from ARPES data and FT STS maps at $-50 \mathrm{meV}$ of the two terminations. (a) and (b) are the schematics of the spin texture derived from the $k \cdot p$ Hamiltonian for the QL (a) and SL (b) terminations. Arrows and colors of the vectors represent the in-plane (purple) and out-of-plane (red, $+z$; blue, $-z$ ) components of spin at the contour maps of the Fermi energy, respectively. The black arrow indicates the $\bar{\Gamma}-\bar{M}$ direction for all the maps. For QL termination, the hexagonal warping effect causes a canted spin at the $z$ direction. For SL termination, a pair of Rashba-like splitting bands contribute antiparallel helicity for the inner and outer rings in the CCE map. A strong warping effect is observed as a flowershaped band in between the rings. (c),(d) CCE maps from ARPES measurements at 50 meV below Fermi level for QL (c) and SL (d) terminations. (e),(f) JDOS calculated from the intensity of the CCE map are shown for QL (e) and SL (f) terminations. For the calculation on the SL, idealized bands with an experimentally determined adjusted intensity ratio are used. The JDOS maps show unexpected scattering intensity at $\bar{\Gamma}-\bar{K}$ directions. (g),(h) Taking into account the spin-selective joint density of states, the scattering maps are (g) for QL and (h) SL terminations. For both simulations, the scattering at $\bar{\Gamma}-\bar{K}$ directions are greatly suppressed. (i) and (j) are the Fourier transformation on the $d I / d V$ maps (FT STS) at $V_{\text {bias }}=-50 \mathrm{mV}, I_{t}=500 \mathrm{pA}$, image size $100 \times 100 \mathrm{~nm}^{2}$, showing good agreement with the spin-selective JDOS maps. The scale bars are $0.1 \AA^{-1}$ in CCE maps and $0.2 \AA^{-1}$ in the rest maps. FT STS maps are rotationally symmetrized.

hybridization strength between the top two layers, while $\tau$ and $\boldsymbol{\sigma}$ are Pauli matrices with $\tau_{ \pm}=\left(1 \pm \tau_{z}\right) / 2$. The corresponding surface band dispersions of both terminations, calculated from Eq. (1), reproduce a direct gap in the QL termination due to the combined effect of the hybridization $\Delta$ and the exchange field $m_{z}^{R}$, as well as a gapless Dirac cone beneath the Rashba bands [see Figs. S12 (a) and S12(c) [32] ]. We next use the obtained spin textures to calculate the spin-selective JDOS and compare with the observed QPI.

The spin configurations derived from the $k \cdot p$ model near the Fermi level are summarized in Figs. 3(a) and 3(b). We find that (i) the QL termination carries a spin-helical Dirac state with hexagonal warping, which gives rise to the canted spin texture along the $z$ direction; (ii) on the SL termination, a Rashba-like splitting state comprises a pair of concentric circular bands with antiparallel spin helicity, between which lies another strongly canted helical state whose spin is antiparallel to the outer ring. The spinselective JDOS can be estimated by adding a spin scattering matrix to the JDOS calculation via $\operatorname{SSJDOS}(\boldsymbol{q}, E)=$ $\int I(\boldsymbol{k}, E) T(\boldsymbol{q}, \boldsymbol{k}, E) I(\boldsymbol{k}+\boldsymbol{q}, E) d^{2} \boldsymbol{k}, \quad$ where $T(\boldsymbol{q}, \boldsymbol{k}, E)=$ $|\langle\boldsymbol{S}(\boldsymbol{k}, E) \mid \boldsymbol{S}(\boldsymbol{k}+\boldsymbol{q}, E)\rangle|^{2} \quad$ [27] describes the scattering matrix where only the states with overlapped spin can be scattered. By assigning the above-mentioned spin configuration [Figs. 3(a) and 3(b)] to the CCE maps of ARPES [Figs. 3(c) and 3(d)], the spin-selective JDOS maps at $E=-50 \mathrm{meV}$ are calculated in Figs. 3(g) and 3(h). For the QL termination, there is a remarkable similarity between the QPI map [Fig. 3(i)] and spin-selective JDOS [Fig. 3(g)]. Compared with the JDOS map [Fig. 3(e)], the scattering intensity along $\bar{\Gamma}-\bar{K}$ directions is greatly suppressed, confirming the previously discussed hexagonal warping effect. For the SL termination, the QPI map is with a sixpetal flower shape. Similar to the QL termination, there is clear attenuation of $\bar{\Gamma}-\bar{K}$ scattering, while the $\bar{\Gamma}-\bar{M}$ scattering is preserved, if we compare the QPI map [Fig. 3(j)] with the JDOS map [Fig. 3(f)]. The decreased scattering intensity along $\bar{\Gamma}-\bar{K}$ directions is well captured by the spin-selective JDOS map in Fig. 3(h), caused by the helicity assignment in the Rashba-like splitting bands (inner and outer rings) and the strongly warped band. Moreover, it is worth noting that, in the ARPES CCE map on the SL [Fig. 3(d)], a small hybridization gap appears near the Fermi level when the strongly warped band and the outer circular band intersect along $\bar{\Gamma}-\bar{M}$ directions. Such spin texture 

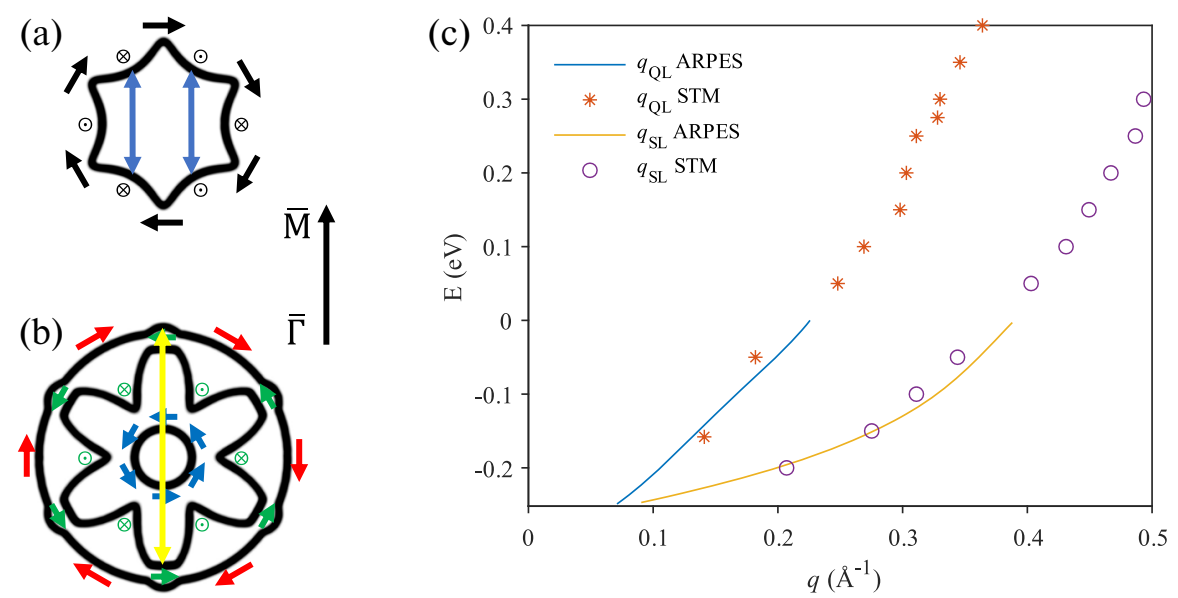

FIG. 4. Dispersion of $\Gamma-M$ scattering edges from ARPES and FT STS. (a),(b) Schematic of CCEs resulting in the edges of $q$ vectors observed in the FT STS maps along the $\bar{\Gamma}-\bar{M}$ direction; arrows mark the spin orientation on these topological surface states: on the QL termination (a), the circular shape surface band is slightly warped for $E>-100 \mathrm{meV}$, causing a canted spin along $z$, thus resulting in $q_{\mathrm{QL}}$ marked by the blue arrows; on the SL termination (b), for $E>-150 \mathrm{meV}$, Rashba-like spin splitting contributes antiparallel helicity for the inner and outer rings in the CCE map (marked as blue and red arrows), where the outer ring hybridizes with a strongly warped surface band sandwiched in between. The middle warped band has the same helicity as the inner circular bands to fit the intensity along the $\bar{\Gamma}-\bar{M}$ direction in the QPI map, leading to $q_{\mathrm{SL}}$ marked by the yellow arrow. (c) Dispersion of the $q$ vectors calculated from the ARPES data (solid lines) on the QL (blue) and SL (yellow) terminations and the $q$ vectors obtained from the FT STS data (orange stars for QL and purple circles for SL). The original FT STS and CCE maps are shown in Figs. S6-S9 [32], respectively.

from the hybridization allows scattering around $\bar{\Gamma}-\bar{M}$ directions with the largest wave vectors [see the yellow arrow in Fig. 4(b)], preserving the scattering intensity along the curved edges of the petals, as seen in Fig. 3(h). On the other hand, the calculated spin-selective JDOS map show little intensity inside the petals compared with the QPI map. The rearranged spin texture near hybridization between the outer circular band and the strongly warped band could be more complicated than the simplified model we use for the simulation, which could result in redistributed scattering intensity at the six petals. Scattering from other energy cuts may also play a role. However, despite the inevitable discrepancy resulting from a simplified model, the strong correlation between the QPI and spin-selective JDOS confirms the topological nature of the surface bands as well as the spin configurations on the two distinct terminations of $\mathrm{MnBi}_{4} \mathrm{Te}_{7}$, highlighting the hybridization between the strongly canted helical state from the surface SL and the Rashba-like splitting states from its neighboring QL.

Consequently, as illustrated by Figs. 4(a) and 4(b), the major scattering channel forming the edge of the petals in the QPI pattern can be originated only from $q_{\mathrm{SL}}$ along $\bar{\Gamma}-\bar{M}$ with aligned spins for the SL termination, while for QL termination, the majority of allowed scattering occurs for wave vector $q_{\mathrm{QL}}$. We then overlay the dispersion of the wave vectors $q_{\mathrm{QL}}$ and $q_{\mathrm{SL}}$ obtained in the QPI patterns (Figs. S6, S7, and S11 [32]) with the energy dispersion expected in the spin-selective JDOS calculated from ARPES data (Figs. S8 and S9 [32]). The plot [Fig. 4(c)] shows good agreement between QPI and ARPES below the Fermi level for both terminations, reassuring the topological nature of these bands. The dispersion for both vectors remains at the high-energy regime up to $400 \mathrm{meV}$ (about $685 \mathrm{meV}$ above the Dirac point), showing robust surface states.

\section{CONCLUSION}

In summary, we have studied the distinct topological surface states of the two terminations of $\mathrm{MnBi}_{4} \mathrm{Te}_{7}$ by ARPES and STM. Compared with theoretical calculations, a gapless Dirac electronic structure is observed on the SL termination, whereas the QL termination is found to be gapped because of the hybridization between different orbitals of the neighboring TI building blocks, implying different surface magnetism on the two terminations. Despite the microscopic defects related to the magnetic atoms, the scattering process on both terminations can be understood by the topological surface states and the newly discovered spin configurations. The robust topological surface states remain present up to an energy regime far above the Fermi energy on both terminations, showing great tolerance to disorders. Our results provide insight not only to the impact of surface magnetism on the topological surface states, but also the potential of the hybridization effect in the heterostructure engineering in magnetic TI systems. Further surface-sensitive magnetic measurements or quantum transport study may be able to directly probe the detailed surface magnetic ordering on the two terminations and the related exotic quantum states. 


\section{ACKNOWLEDGMENTS}

The research was supported by National Natural Science Foundation of China under Projects No. 11674150, No. 11674149, No. 11874195, and No. 11804402, the Key-Area Research and Development Program of Guangdong Province (2019B010931001), Guangdong Innovative and Entrepreneurial Research Team Program (2016ZT06D348 and 2017ZT07C062), the Guangdong Provincial Key Laboratory of Computational Science and Material Design (Grant No. 2019B030301001), the Science, Technology, and Innovation Commission of Shenzhen Municipality (JCYJ20150630145302240, G02206304, and G02206404), and Center for Computational Science and Engineering at SUSTech. M.X. acknowledges the financial support from a Collaborative Research Fund (C7036-17W) from the Research Grant Council, Hong Kong Special Administrative Region. C. L. acknowledges support from the Highlight Project (No. PHYS-HL-2020-1) of the College of Science, SUSTech. ARPES experiments were performed with the approval of the Hiroshima Synchrotron Radiation Center (HSRC), Hiroshima, Japan under Proposals No. 19AU004, No. 19AU015, and No. 19AG002. We thank Jianpeng Liu, Haizhou Lu, and Junhao Lin for valuable discussions.

\section{APPENDIX: METHODS}

Single crystals of $\mathrm{MnBi}_{4} \mathrm{Te}_{7}$ are prepared by the flux growth method and confirmed by single crystal x-ray diffraction data and the subsequent magnetic and transport measurements. The crystal has an AFM ground state with $T_{N} \sim 12 \mathrm{~K}$ and a critical field of the spin-flop transition of $B_{c}=0.2 T$, as shown in Fig. S1 [32]. The STM measurements are performed on in situ cleaved surfaces of $\mathrm{MnBi}_{4} \mathrm{Te}_{7}$ using commercialized STM (Unisoku 1500) operating at approximately $4.5 \mathrm{~K}$ with a base pressure better than $1 \times 10^{-10}$ mbar. Tungsten tips are modified in situ by scanning on copper when needed. The $d I / d V$ spectroscopy is obtained by a standard lock-in technique with frequency $687.2 \mathrm{~Hz}$ and an ac modulation of $5 \mathrm{mV}$. The spatial resolution for the FT STS maps is $0.17 \mathrm{~nm}$. For the QL termination, the JDOS data are calculated by the self-convolution of raw CCE from ARPES, and a scattering matrix taking into account of the spin texture derived from the $k \cdot p$ model (Fig. S12 [32]) is used for the spin-selective JDOS. For the SL termination, an idealized CCE constructed based on the ARPES CCE is used for the JDOS and spin-selective JDOS calculation because of the complexity of the spin configuration in the topological bands. Details can be found in Supplemental Material [32].

ARPES measurements are performed at the Hiroshima Synchrotron Radiation Center (HiSOR). The spin-integrated spectra are obtained with an offline ARPES setup under $6.36 \mathrm{eV}$ laser light, while the spin-resolved ARPES map
[Fig. 2(d)] is obtained at Beam line 9B of HiSOR, using a VLEED spin detector and $18 \mathrm{eV}$ incident light.

The surface band structures of both terminations are calculated by density functional theory. We use the projector-augmented wave (PAW) pseudopotentials [49] with the exchange correlation of Perdew-Burke-Ernzerhof (PBE) form [50] and GGA $+U$ approach within the Dudarev scheme [51] as implemented in the Vienna $A b$ Initio Simulation Package [52,53]. The energy cutoff is chosen 1.5 times as large as the values recommended in relevant pseudopotentials. The $U$ value is set to be $5 \mathrm{eV}$. The $k$-points-resolved value of BZ sampling is $0.02 \times 2 \pi \AA^{-1}$. The total energy minimization is performed with a tolerance of $10^{-6} \mathrm{eV}$. Spin-orbit coupling is included self-consistently throughout the calculations. The surface band structures of QL and SL terminations are obtained from slab calculations with the thickness of 9 and 7 van der Waals layers, respectively.

[1] Y. Tokura, K. Yasuda, and A. Tsukazaki, Magnetic Topological Insulators, Nat. Rev. Phys. 1, 126 (2019).

[2] C.-Z. Chang et al., Experimental Observation of the Quantum Anomalous Hall Effect in a Magnetic Topological Insulator, Science 340, 167 (2013).

[3] R. Yu, W. Zhang, H.-J. Zhang, S.-C. Zhang, X. Dai, and Z. Fang, Quantized Anomalous Hall Effect in Magnetic Topological Insulators, Science 329, 61 (2010).

[4] Y. Gong et al., Experimental Realization of an Intrinsic Magnetic Topological Insulator, Chin. Phys. Lett. 36, 076801 (2019).

[5] J. Li, Y. Li, S. Du, Z. Wang, B.-L. Gu, S.-C. Zhang, K. He, W. Duan, and Y. Xu, Intrinsic Magnetic Topological Insulators in van der Waals Layered $\mathrm{MnBi}_{2} \mathrm{Te}_{4}$-Family Materials, Sci. Adv. 5, eaaw5685 (2019).

[6] D. Zhang, M. Shi, T. Zhu, D. Xing, H. Zhang, and J. Wang, Topological Axion States in the Magnetic Insulator $\mathrm{MnBi}_{2} \mathrm{Te}_{4}$ with the Quantized Magnetoelectric Effect, Phys. Rev. Lett. 122, 206401 (2019).

[7] M. M. Otrokov et al., Prediction and Observation of an Antiferromagnetic Topological Insulator, Nature (London) 576, 416 (2019)

[8] E. D. L. Rienks et al., Large Magnetic Gap at the Dirac Point in $\mathrm{Bi}_{2} \mathrm{Te}_{3} / \mathrm{MnBi}_{2} \mathrm{Te}_{4}$ heterostructures, Nature (London) 576, 423 (2019)

[9] M. M. Otrokov, I. P. Rusinov, M. Blanco-Rey, M. Hoffmann, A. Yu. Vyazovskaya, S. V. Eremeev, A. Ernst, P. M. Echenique, A. Arnau, and E. V. Chulkov, Unique Thickness-Dependent Properties of the van der Waals Interlayer Antiferromagnet $\mathrm{MnBi}_{2} \mathrm{Te}_{4}$ Films, Phys. Rev. Lett. 122, 107202 (2019)

[10] H. Sun, B. Xia, Z. Chen, Y. Zhang, P. Liu, Q. Yao, H.Tang, Y. Zhao, H. Xu, and Q. Liu, Rational Design Principles of the Quantum Anomalous Hall Effect in Superlatticelike Magnetic Topological Insulators, Phys. Rev. Lett. 123, 096401 (2019)

[11] Y. Deng, Y. Yu, M. Z. Shi, Z. Guo, Z. Xu, J. Wang, X. H. Chen, and Y. Zhang, Quantum Anomalous Hall Effect in 
Intrinsic Magnetic Topological Insulator $\mathrm{MnBi}_{2} \mathrm{Te}_{4}$, Science 367, 895 (2020).

[12] J. Ge et al., High-Chern-Number and High-Temperature Quantum Hall Effect without Landau Levels, arXiv:1907 .09947 [Natl. Sci. Rev. (to be published)].

[13] C. Liu, Y. Wang, H. Li, Y. Wu, Y. Li, J. Li, K. He, Y. Xu, J. Zhang, and Y. Wang, Robust Axion Insulator and Chern Insulator Phases in a Two-Dimensional Antiferromagnetic Topological Insulator, Nat. Mater. 19, 522 (2020)

[14] Y.-J. Hao et al., Gapless Surface Dirac Cone in Antiferromagnetic Topological Insulator $\mathrm{MnBi}_{2} \mathrm{Te}_{4}$, Phys. Rev. X 9 , 041038 (2019).

[15] H. Li et al., Dirac Surface States in Intrinsic Magnetic Topological Insulators $\mathrm{EuSn}_{2} \mathrm{As}_{2}$ and $\mathrm{MnBi}_{2 n} \mathrm{Te}_{3 n+1}$, Phys. Rev. X 9, 041039 (2019).

[16] Y. J. Chen et al., Topological Electronic Structure and Its Temperature Evolution in Antiferromagnetic Topological Insulator $\mathrm{MnBi}_{2} \mathrm{Te}_{4}$, Phys. Rev. X 9, 041040 (2019).

[17] P. Swatek, Y. Wu, L.-L. Wang, K. Lee, B. Schrunk, J. Yan, and A. Kaminski, Gapless Dirac Surface States in the Antiferromagnetic Topological Insulator $\mathrm{MnBi}_{2} \mathrm{Te}_{4}$, Phys. Rev. B 101, 161109 (2020).

[18] R. C. Vidal et al., Topological Electronic Structure and Intrinsic Magnetization in $\mathrm{MnBi}_{4} \mathrm{Te}_{7}: A \mathrm{Bi}_{2} \mathrm{Te}_{3}$ Derivative with a Periodic Mn Sublattice, Phys. Rev. X 9, 041065 (2019).

[19] J. Wu et al., Natural van der Waals Heterostructural Single Crystals with Both Magnetic and Topological Properties, Sci. Adv. 5, eaax9989 (2019).

[20] X.-M. Ma et al., Hybridization-Induced Gapped and Gapless States on the Surfaces of Magnetic Topological Insulators, arXiv:1912.13237.

[21] Y. Hu et al., Universal Gapless Dirac Cone and Tunable Topological States in $\left(\mathrm{MnBi}_{2} \mathrm{Te}_{4}\right)_{m}\left(\mathrm{Bi}_{2} \mathrm{Te}_{3}\right)_{n}$ Heterostructures, Phys. Rev. B 101, 161113 (2020).

[22] L.X. Xu et al., Persistent Gapless Surface States in $\mathrm{MnBi}_{2} \mathrm{Te}_{4} / \mathrm{Bi}_{2} \mathrm{Te}_{3} \quad$ Superlattice Antiferromagnetic Topological Insulator, arXiv:1910.11014.

[23] C. Hu et al., A van der Waals Antiferromagnetic Topological Insulator with Weak Interlayer Magnetic Coupling, Nat. Commun. 11, 97 (2020).

[24] C. Hu et al., Realization of an Intrinsic, Ferromagnetic Axion Insulator in $\mathrm{MnBi}_{8} \mathrm{Te}_{13}$, arXiv:1910.12847.

[25] K. N. Gordon et al., Strongly Gapped Topological Surface States on Protected Surfaces of Antiferromagnetic $\mathrm{MnBi}_{4} \mathrm{Te}_{7}$ and $\mathrm{MnBi}_{6} \mathrm{Te}_{10}$, arXiv:1910.13943.

[26] M. Z. Hasan and C. L. Kane, Colloquium: Topological Insulators, Rev. Mod. Phys. 82, 3045 (2010).

[27] P. Roushan, J. Seo, C. V. Parker, Y. S. Hor, D. Hsieh, D. Qian, A. Richardella, M.Z. Hasan, R. J. Cava, and A. Yazdani, Topological Surface States Protected from Backscattering by Chiral Spin Texture, Nature (London) 460 , 1106 (2009).

[28] Y. Okada et al., Direct Observation of Broken TimeReversal Symmetry on the Surface of a Magnetically Doped Topological Insulator, Phys. Rev. Lett. 106, 206805 (2011).

[29] T. Zhang et al., Experimental Demonstration of Topological Surface States Protected by Time-Reversal Symmetry, Phys. Rev. Lett. 103, 266803 (2009).
[30] Y. Yuan et al., Electronic States and Magnetic Response of $\mathrm{MnBi}_{2} \mathrm{Te}_{4}$ by Scanning Tunneling Microscopy and Spectroscopy, arXiv:2001.00462v1.

[31] Z. Liang et al., Mapping the Dirac Fermions in Intrinsic Antiferromagnetic Topological Insulators $\left(\mathrm{MnBi}_{2} \mathrm{Te}_{4}\right)$ $\left(\mathrm{Bi}_{2} \mathrm{Te}_{3}\right)_{n}(n=0,1)$, arXiv:2001.00866v1.

[32] See Supplemental Material at http://link.aps.org/ supplemental/10.1103/PhysRevX.10.031013 for Supplemental Figures and the algorithm for calculating JDOS and spin-selective JDOS maps.

[33] J.-Q. Yan, Q. Zhang, T. Heitmann, Z. Huang, K. Y. Chen, J.G. Cheng, W. Wu, D. Vaknin, B. C. Sales, and R. J. McQueeney, Crystal Growth and Magnetic Structure of $\mathrm{MnBi}_{2} \mathrm{Te}_{4}$, Phys. Rev. Mater. 3, 064202 (2019).

[34] Y.S. Hor et al., Development of Ferromagnetism in the Doped Topological Insulator $\mathrm{Bi}_{2-x} \mathrm{Mn}_{x} \mathrm{Te}_{3}$, Phys. Rev. B 81, 195203 (2010).

[35] H. Beidenkopf, P. Roushan, J. Seo, L. Gorman, I. Drozdov, Y. S. Hor, R. J. Cava, and A. Yazdani, Spatial Fluctuations of Helical Dirac Fermions on the Surface of Topological Insulators, Nat. Phys. 7, 939 (2011).

[36] Y. L. Chen et al., Massive Dirac Fermion on the Surface of a Magnetically Doped Topological Insulator, Science 329, 659 (2010).

[37] I. Lee et al., Imaging Dirac-Mass Disorder from Magnetic Dopant Atoms in the Ferromagnetic Topological Insulator $\mathrm{Cr}_{x}\left(\mathrm{Bi}_{0.1} \mathrm{Sb}_{0.9}\right)_{2-x} \mathrm{Te}_{3}$, Proc. Natl. Acad. Sci. U.S.A. 112, 1316 (2015).

[38] H. Iwasawa, E. F. Schwier, M. Arita, A. Ino, H. Namatame, M. Taniguchi, Y. Aiura, and K. Shimada, Development of Laser-Based Scanning $\mu$-ARPES System with Ultimate Energy and Momentum Resolutions, Ultramicroscopy 182, 85 (2017).

[39] J.-Q. Yan et al., A-type Antiferromagnetic Order in $\mathrm{MnBi}_{4} \mathrm{Te}_{7}$ and $\mathrm{MnBi}_{6} \mathrm{Te}_{10}$ Single Crystals, Phys. Rev. Mater. 4, 054202 (2020).

[40] B. Niu et al., Coexistence of Magnetic Orders in TwoDimensional Magnet $\mathrm{CrI}_{3}$, Nano Lett. 20, 553 (2020).

[41] F. Yang, Y. R. Song, H. Li, K. F. Zhang, X. Yao, C. Liu, D. Qian, C. L. Gao, and J.-F. Jia, Identifying Magnetic Anisotropy of the Topological Surface State of $\mathrm{Cr}_{0.05} \mathrm{Sb}_{1.95} \mathrm{Te}_{3}$ with Spin-Polarized STM, Phys. Rev. Lett. 111, 176802 (2013).

[42] A. Chikina et al., Strong Ferromagnetism at the Surface of an Antiferromagnet Caused by Buried Magnetic Moments, Nat. Commun. 5, 3171 (2014).

[43] M. F. Crommie, C. P. Lutz, and D. M. Eigler, Imaging Standing Waves in a Two-Dimensional Electron Gas, Nature (London) 363, 524 (1993).

[44] J. E. Hoffman, K. McElroy, D.-H. Lee, K. M. Lang, H. Eisaki, S. Uchida, and J. C. Davis, Imaging Quasiparticle Interference in $\mathrm{Bi}_{2} \mathrm{Sr}_{2} \mathrm{CaCu}_{2} \mathrm{O}_{8+\delta}$, Science 297, 1148 (2002).

[45] Z. Alpichshev, J. G. Analytis, J.-H. Chu, I. R. Fisher, Y. L. Chen, Z.X. Shen, A. Fang, and A. Kapitulnik, STM Imaging of Electronic Waves on the Surface of $\mathrm{Bi}_{2} \mathrm{Te}_{3}$ : Topologically Protected Surface States and Hexagonal Warping Effects, Phys. Rev. Lett. 104, 016401 (2010). 
[46] T. Okuda, K. Miyamoto, A. Kimura, H. Namatame, and M. Taniguchi, A Double VLEED Spin Detector for HighResolution Three Dimensional Spin Vectorial Analysis of Anisotropic Rashba Spin Splitting, J. Electron Spectrosc. Relat. Phenom. 201, 23 (2015).

[47] M.S. Bahramy et al., Emergent Quantum Confinement at Topological Insulator Surfaces, Nat. Commun. 3, 1159 (2012).

[48] L. Fu, Hexagonal Warping Effects in the Surface States of the Topological Insulator $\mathrm{Bi}_{2} \mathrm{Te}_{3}$, Phys. Rev. Lett. 103, 266801 (2009).

[49] G. Kresse and D. Joubert, From Ultrasoft Pseudopotentials to the Projector Augmented-Wave Method, Phys. Rev. B 59, 1758 (1999).
[50] J. P. Perdew, K. Burke, and M. Ernzerhof, Generalized Gradient Approximation Made Simple, Phys. Rev. Lett. 77, 3865 (1996).

[51] S. L. Dudarev, G. A. Botton, S. Y. Savrasov, C. J. Humphreys, and A. P. Sutton, Electron-Energy-Loss Spectra and the Structural Stability of Nickel Oxide: An LSDA $+U$ study, Phys. Rev. B 57, 1505 (1998).

[52] G. Kresse and J. Furthmüller, Efficiency of Ab-Initio Total Energy Calculations for Metals and Semiconductors Using a Plane-Wave Basis Set, Comput. Mater. Sci. 6, 15 (1996).

[53] G. Kresse and J. Furthmüller, Efficient Iterative Schemes for Ab Initio Total-Energy Calculations Using a Plane-Wave Basis Set, Phys. Rev. B 54, 11169 (1996). 